\title{
Resignificación de la práctica pedagógica desde la perspectiva de inclusión educativa
}

\author{
Resignifying teaching practice from the perspective of \\ inclusive education
}

\author{
Nayibis Cano Acevedo, nayicano21@hotmail.es \\ María Claudia Ramos Espitia,laprofe_ramos@hotmail.com \\ Universidad de Cartagena, Colombia
}

\section{Resumen}

La inclusión es más que acceso, implica minimizar las barreras que impiden la participación y el aprendizaje. Este estudio de caso interpretativo analiza las posibilidades de resignificación de las prácticas pedagógicas de los maestros con la mediación de las didácticas flexibles basadas en el Diseño Universal para el Aprendizaje (DUA). Para ello, busca develar nuevas posibilidades de enseñar en contextos de vulnerabilidad social atendiendo a la diversidad como valor que enriquece el proceso educativo. Ante una educación homogeneizante e igualitaria que no atiende a la diferencia, se procura por una propuesta educativa en la que se aprenda no solo a vivir y aprender de las diferencias. Los resultados evidencian que persisten en los maestros imaginarios que presentan la diversidad como sinónimo de dificultad que entorpece el proceso, ya que la pedagogía que emplean se basa en modelos tradicionales de enseñanza que invisibilizan la diferencia y se da un trato igualitario para todos los aprendices, además consideran no estar suficientemente preparados para abordar la diversidad desde el aula. A partir de un proceso de reflexión sobre su quehacer, los maestros reconocen la necesidad de una práctica resignificada que facilite el desarrollo humano de los aprendices en todas sus dimensiones y potencialice al máximo las capacidades para aprender desde el despliegue de múltiples formas para enseñar, destacándose la necesidad de capacitación continua sobre inclusión educativa y el interés por articular su práctica actual con las didácticas flexibles.

Palabras claves: Diferencia, diversidad, equidad, igualdad, participación, reflexión.

\section{Abstract}

Inclusion is more than access, for it involves minimizing the barriers to participation and learning. This research analyzes the possibilities of resignifying teaching practices with the mediation of the flexible didactics based on the Universal Design for Learning (UDL). The study seeks to reveal new possibilities of teaching in contexts of social vulnerability, attending to diversity as a value that enriches educational processes. In the face of a homogenizing and equal education that does not attend to the differences, it is sought an educational proposal in which it is learned to live not only with the differences, but also to learn of them. Results show that teachers still conceive diversity as a synonym for difficulty that impedes the educational process, since teachers' methodology is based on traditional teaching models that obscure the differences and gives an equal treatment to all learners. Teachers also consider they are not trained enough to address diversity in the classroom. Based on reflection on their work and on the use of multiple ways of teaching, teachers recognize the need to give new meaning to their educational practice, facilitating learners' human development and making the most of their learning abilities. Findings also highlight the need for a continuous training on inclusive education and the interest on articulating teachers' current practices with flexible didactics.

Keywords: Difference, diversity, equity, equality, participation, reflection. 


\section{Introducción}

Alcanzar una educación de calidad ha sido el propósito de políticas internacionales, nacionales, regionales y locales, por ende, ha sido un tema de gran importancia abordado desde la agenda gubernamental y el Ministerio de Educación Nacional (MEN) de Colombia, con especial énfasis en los últimos años en el proceso de inclusión educativa, entendida como la posibilidad de que todos, sin ningún distingo, accedan a una educación de calidad que dé respuesta a la diversidad inherente a cada ser. Con ello se pretende derribar cualquier barrera para el aprendizaje y la participación, ya que la respuesta educativa actual no es acorde con las necesidades, intereses y expectativas de los estudiantes. Según la Organización de las Naciones Unidas para la Educación, la Ciencia y la Cultura (Unesco, 2005), la inclusión es una «estrategia dinámica para responder en forma proactiva a la diversidad de los estudiantes y concebir las diferencias individuales no como problema sino como oportunidades para enriquecer el aprendizaje» (p. 11). Por tanto, es la escuela la que debe adecuarse a la diversidad y estar preparada para asumir la diferencia como la norma y no como la excepción, ya que, como indica Blanco (2006), «muchos estudiantes experimentan dificultades de aprendizaje y de participación en las escuelas como consecuencia de la rigidez y la homogeneidad de la respuesta educativa» (p. 15).

Al hablar de inclusión educativa, decimos que todos cabemos en un mismo espacio facilitador de la construcción de saberes que permite crecer juntos, aprender juntos, vivir juntos, hacer juntos. En tal sentido, de acuerdo con Delors (1996), se trata de entender que «la educación puede ser un factor de cohesión social si procura tener en cuenta la diversidad de los individuos y de los grupos humanos y al mismo tiempo, evitar ser a su vez un factor de exclusión social» (p. 59). Así entendida, la diversidad es un valor que ayuda a la unión de los grupos sociales permitiendo cerrar brechas que social, cultural e históricamente el mismo hombre ha creado.

Concebir la escuela como un espacio donde no se generen prácticas discriminatorias que atenten contra la libertad individual de los estudiantes y sea posible la premisa anterior, requiere que la respuesta educativa no sea homogeneizante sino que se reoriente desde la heterogeneidad. Cuando las prácticas pedagógicas enfatizan en contenidos y no en la construcción conjunta de saberes y dejan de lado la importancia de atender a los estilos y ritmos de aprendizaje como posibilidad para ayudar al crecimiento del otro, vulneran la participación porque que se atiende a estudiantes que se sienten integrados pero no incluidos. En otras palabras, los estudiantes siguen estando excluidos a pesar de estar inmersos en un contexto escolar. Por ello, como lo advierte Ortiz (2000), «es la escuela la que debe estar pensada y preparada para responder a las características de todos los estudiantes, partiendo de que todos somos básicamente diferentes, sean cuales sean las circunstancias personales y sociales» (p. 5).

El tema de la inclusión educativa ha sido objeto de múltiples estudios en el ámbito internacional, nacional y local, pero se ha enfocado en la inclusión de los niños con necesidades educativas especiales (NEE), ya que históricamente las dificultades de aprendizaje han sido atribuidas a variables relacionadas con el propio individuo, no evidenciando que lo educativo, familiar y social tienen gran influencia en el desarrollo y aprendizaje de las personas. Bien lo anotan Booth y Ainscow (2000), cuando advierten que «las barreras para aprender y participar surgen a través de una interacción entre los estudiantes y su contexto, las personas, políticas, instituciones, culturas y circunstancias sociales y económicas que afectan sus vidas» (p. 45). Así, se espera que sean los estudiantes quienes se adapten a la escuela, independientemente de sus características particulares.

Esta realidad educativa común a nivel general, se da en la Institución Educativa Ana María Vélez de Trujillo sede Loma Fresca, pues la educación actual continúa encajonada en un enfoque 
tradicional en donde se privilegian los resultados por encima de los procesos mismos de construcción del conocimiento. Se pone en lugar de privilegio la repetición de conceptos y el abordaje de contenidos establecidos de manera previa por maestros, ciñéndose a un currículo rígido que deja poco margen al desarrollo creativo de unas prácticas de aula centradas en el aprendiz. Persiste la figura del maestro como aquel que posee el conocimiento y por ende el control de cada actividad, proceso y verificación de resultados. Cuando el aprendiz no actúa en consonancia con estos parámetros se considera con dificultades, las que en todo caso se atribuyen a él mismo.

La población estudiantil de la sede Loma Fresca es diversa, heterogénea, con marcadas diferencias de raza, credo, cultura y condiciones socioeconómicas, por cuanto hay población que ha sido objeto de desplazamiento por situaciones de conflicto y violencia. Sin embargo, los estudiantes son acogidos en la escuela sin distingos, lo que no quiere decir que sean atendidos de acuerdo a sus características y necesidades particulares. Todos acceden a una educación igualitaria y sin distinción, pero esta limita a la participación, al no tener en cuenta sus individualidades y particularidades.

Todo lo expuesto deriva en un ambiente poco favorable para que se den intercambios gratificantes entre el contenido presentado por el maestro y lo que efectivamente es asimilado por el estudiante. A propósito de la evolución del rol que desempeñan el docente, Gardner (1983), anota:

El docente debe repensar su práctica y apostarle al diseño, planeación y desarrollo de prácticas educativas inclusivas que superen las barreras del tiempo, los recursos y los espacios y que tuvieran en cuenta a todos los niños y niñas e intentaran responder a sus estilos de trabajo (p. 243).

Al respecto resulta pertinente preguntarse: ¿Cómo resignificar la práctica pedagógica actual llevada a cabo por los maestros de la Institución Educativa Ana María Vélez de Trujillo sede Loma Fresca mediada por las didácticas flexibles para favorecer la inclusión educativa? La presente investigación pretende contribuir a la promoción de prácticas pedagógicas mediadas por didácticas flexibles que favorezcan la inclusión educativa desde la preocupación reflexiva de sus actores y a partir del análisis de cómo se está llevando a cabo dicha práctica. A partir de allí se pretende derivar modos diferentes de enseñar en procura de alcanzar algo distinto para los aprendices en el reconocimiento de que todos pueden aprender, visibilizando a aquellos que requieran de un mayor apoyo.

Para contribuir al logro de mejores aprendizajes es necesario que el maestro considere el contexto sociocultural del estudiante. La educación, y por ende la práctica que el maestro ejerce, no puede estar de espaldas a la realidad que afecta la vida de los aprendices. Esta consideración permitirá una mayor y mejor atención del aprendiz en concordancia con su realidad, todo a través de la definición de criterios comunes que permeen la dinámica escolar.

El Diseño Universal para el Aprendizaje (DUA), desde la inclusión propicia y da respuesta a todo tipo de estudiantes, independientemente de sus capacidades, conocimientos, procedencia o experiencias personales. El Center for Applied Special Technology (2011), indica que es considerado para el contexto educativo como uno de los enfoques más prometedores pues posibilita un aprendizaje que atiende básicamente a tres principios: «Múltiples medios de Representación (el qué del aprendizaje), Múltiples medios para la Acción y la Expresión (el cómo del aprendizaje) ${ }^{2}$, Múltiples Medios de Compromiso (el porqué del aprendizaje)» (p. 28). Cada principio obedece al deseo de dar cabida a la participación y la acción de los aprendices, haciendo del conocimiento algo más atrayente para ellos. En síntesis, como lo advierten, el enfoque se preocupa porque «todos los alumnos puedan acceder a los contenidos y objetivos del currículo ordinario» (p. 879). 


\section{Marco teórico}

La práctica pedagógica es el escenario donde el maestro dispone de todos aquellos elementos propios de su competencia académica y personal a la hora de enseñar: desde lo académico, lo relacionado con su saber disciplinar; desde lo pedagógico, lo referente a la reflexión continua que haga acerca de las fortalezas y debilidades implícitas en su quehacer. Para Vygotsky (1998), se debe concebir el espacio educativo como un lugar de potencialidades de aprendizaje y a las prácticas educativas como realidades sujetas a significación y resignificación. Por tanto, la práctica pedagógica es un saber en cuestión que demanda del maestro -como sujeto del saber- reflexión continua y permanente, enfatizando en su dimensión didáctica. Tal ejercicio le permite analizar la forma como orienta el proceso de enseñanza.

Para este estudio se considera la dimensión didáctica como aquella desde donde es posible entrar a accionar en el aula, pues ofrece a los aprendices múltiples modos de aprender. Se considera pertinente su articulación con las didácticas flexibles para facilitar y promover aprendizajes exitosos, atendiendo la diversidad y rompiendo el esquema de un currículo rígido y descontextualizado. Al respecto, como movimiento, la inclusión posibilita aceptar la diversidad como norma y no como excepción. La educación debe apuntar al desarrollo de sistemas educativos inclusivos, como aquellos que asumen la tarea de transformar las escuelas para responder a la diversidad. En ese sentido, la Unesco (2005), define la inclusión como «una estrategia dinámica para responder en forma práctica a la diversidad considerándola no como barrera sino oportunidad para el aprendizaje» (p. 12).

Ante la realidad que acontece y que perpetúa la exclusión bajo el discurso inclusivo, solo será posible alcanzar el logro de aprendizajes más exitosos cuando se asuma la práctica docente como posibilitadora del desarrollo de una mejor dinámica escolar, donde todos los estudiantes se sientan aceptados y acogidos a partir del respeto y atención a la diferencia y la diversidad, y cuando tales aspectos sean acogidos como valores que enriquecen el proceso de enseñanza-aprendizaje. Desde una postura crítica, los maestros deben empezar a cuestionar, transformar, innovar y mejorar la práctica educativa, contribuyendo a su vez a mejorar la calidad educativa.

A la luz de este estudio, las didácticas flexibles son consideradas como un factor clave de éxito para alcanzar la premisa anterior ya que ofrecen nuevas posibilidades de presentación y acercamiento del conocimiento al aprendiz; además, posibilitan que el maestro realice una labor más pertinente desde diferentes modos de enseñar.

El DUA es fundamental para conectar o articular la práctica pedagógica actual con el uso de las didácticas flexibles, pues potencia una educación en y para la vida, donde la participación, la equidad, la permanencia y el acceso son principios rectores; además, la inclusión también debe ser asumida con compromiso y responsabilidad por parte de todos, reconociéndola como pieza fundamental para generar también mejores procesos de convivencia.

El movimiento de la inclusión asume que vivir y aprender juntos es la mejor forma de beneficiar a cualquier persona (Ainscow, 1999; Corbett, 1999; en Moriña, 2004). De allí que es posible generar mejores prácticas de aula si el maestro es consciente de la importancia de autoevaluarse y logra identificar en qué nivel reflexivo se encuentra con relación a la práctica educativa. Esto le permitirá revisar cómo es su relación con sus estudiantes, sus compañeros, la institución y con los valores que desarrolla a través de esta.

Para Larrivee (2000), la práctica pedagógica tiene niveles reflexivos que pueden darse de manera simultánea o no en los maestros, iniciando con un nivel de prerreflexión que puede llegar a la reflexión crítica, donde el maestro es capaz de reflexionar sobre las implicaciones morales y éticas y las consecuencias de sus prácticas de aula con sus estudiantes. Todo proceso reflexivo llevado a cabo por 
los maestros implica la construcción y deconstrucción continua del conocimiento sobre el aprendizaje, el entorno y los procesos educativos; asimismo, también comporta la toma de decisiones sobre la enseñanza misma y sobre cómo se planea y ejecuta la propia práctica, con el fin de transformarla y dotarla de nuevos sentidos.

\section{$1 \quad$ Antecedentes}

Desde la perspectiva de la inclusión educativa, son la escuela y las prácticas pedagógicas las que deben resignificarse para responder a la diversidad. En esa línea, Hopkins y Stern (1996), enfatizan que «los docentes se enfrentan a un reto importante y es cambiar el esquema de homogeneidad por el de heterogeneidad» (p. 501). Diversos estudios dan cuenta de ello, entre los que se destacan algunos que abordan prácticas pedagógicas enmarcadas en acciones inclusivas para dar respuesta a la diversidad.

Por ejemplo, se menciona a Montolio y Cervellera (2008), quienes analizan una escuela que impulsa prácticas escolares inclusivas gestionadas bajo modelos de economía social. Desde esta perspectiva, la diversidad se asume como realidad social que nutre el entramado humano.

Por su parte, López (2008) trata en su estudio la importancia de apoyar procesos que faciliten el desarrollo de comunidades educativas reflexivas, que sean potenciadoras de la educación inclusiva, donde se reduzcan las barreras para la participación y el aprendizaje. Este estudio, llevado a cabo desde una investigación-acción, analiza las posibilidades de que al interior de las escuelas se generen culturas inclusivas atendiendo a poblaciones vulnerables.

A su vez, Rosano (2008), visibiliza en su estudio las condiciones y posibilidades para la educación inclusiva en una comunidad campesina, destacando la práctica pedagógica y la percepción de padres y maestros en lo referente a la educación inclusiva.

En el ámbito nacional, Arias et al. (2007), analizan en su estudio el rol del docente en la escuela tradicional, señalando la importancia de que este transforme su práctica a fin de favorecer la inclusión de los estudiantes con una educación que dé respuesta a la diversidad. Asimismo, Díaz, Franco y Tovar (2008), se fundamentan en las actitudes y percepciones de los docentes hacia la inclusión educativa. Su estudio aporta información valiosa para asegurar el éxito de la inclusión y la calidad educativa.

Se referencian también estudios que además de tratar la inclusión educativa como proceso de gran importancia en la atención a la diversidad, abordan estrategias y didácticas empleadas por los docentes para enseñar, teniendo en cuenta las características particulares de los estudiantes y preocupándose por lograr aprendizajes más significativos y duraderos.

Tal es el caso del estudio realizado por Gisbert y Giné (2011), el cual enfatiza que se debe procurar una formación del profesorado que dé respuesta a la diversidad como tarea colectiva, lo que implica generar y dinamizar ayudas pedagógicas utilizando estrategias como el aprendizaje colaborativo. Con ello se promueve la reflexión pedagógica y la aceptación de la responsabilidad sobre el aprendizaje de los estudiantes.

De otro lado, Arana (2012), da cuenta de cómo hoy día es evidente el desequilibrio marcado entre la teoría y la práctica docente, enfatizando la importancia de que estos reflexionen sobre su práctica a partir de la toma de conciencia acerca de las competencias que desarrollan en su labor, ya que esto repercute en el proceso de enseñanza-aprendizaje.

Mientras que Rodríguez (2013), analiza el nivel de la calidad reflexiva de los profesores y los cambios percibidos en sus prácticas educativas cuando son mediadas por el uso de nuevas estrategias que generan procesos de innovación y cambios para favorecer mejores aprendizajes.

Finalmente, se evidencian experiencias pedagógicas que favorecen los procesos de educación inclusiva procurando la mediación de las didácticas flexibles. Se reseña la investigación realizada 
por Mejía y Molina (2012), la cual determinó la implementación de una estrategia basada en la lúdica y la creatividad, procurando motivar e incentivar a los diferentes actores del proceso educativo a reflexionar acerca de cómo se dan estos procesos a nivel institucional para hacer frente a la diversidad, mirando diferentes formas de ver y hacer educación.

Desde esta óptica la presente investigación procura analizar las posibilidades de resignificación de la práctica pedagógica orientada a atender a la diversidad en el proceso de enseñanza-aprendizaje con la mediación de las didácticas flexibles basadas en el DUA. De esta manera, esta investigación sugiere una posible ruta a seguir para ofrecer a los estudiantes nuevas maneras de aprender a partir del despliegue de nuevas formas de enseñar, resaltando la importancia de que los maestros se preocupen de manera constante por la forma como están llevando el conocimiento a los estudiantes y por las estrategias didácticas que están materializando, de tal forma que la enseñanza sea atrayente, motivadora, generadora de inquietudes, cuestionamientos e intereses que despierten el deseo de los estudiantes por aprender aquello que se le quiere enseñar, reconociendo que construir el aprendizaje con otros resulta una experiencia enriquecedora.

\section{Metodología}

Esta investigación se posiciona desde el enfoque de la investigación cualitativa. En tal sentido, debe ser entendida como una investigación interpretativa donde se estudian los fenómenos y describen los hechos, para luego entenderlos y sacar conclusiones válidas y confiables. Con relación a la investigación cualitativa, Ruiz (1996) observa que,

parte del supuesto básico de que el mundo social es un mundo construido con significados y símbolos, lo que implica la búsqueda de esta construcción y de sus significados (...) entendido siempre en su totalidad, nunca como un fenómeno aislado o fragmentado (p. 217).

La investigación interpretativa en educación se dirige hacia la transformación de la realidad educativa, incluyendo a los protagonistas de esa realidad. Tal investigación procura no solamente producir conocimiento generalizable sino el perfeccionamiento de quienes participan en concreto en cada situación educativa y la transformación de sus conocimientos, actitudes y comportamientos. Así, según Erickson (1986) la investigación interpretativa se convierte en «una modalidad de formación continuada y de transformación institucional en la investigación sobre la enseñanza» ( $p$. 159). Asimismo, Imbernon (1994), indica que «el punto central de la investigación interpretativa es su interés por comprender un fenómeno desde la perspectiva de los participantes de la situación: los actores, y no desde la perspectiva y categoría del investigador» (p. 106).

De la misma forma, en esta investigación se recurrió al estudio de caso por su pertinencia para la comprensión del fenómeno estudiado. A propósito del estudio de caso, Stake (1998) anota que «estudiamos un caso cuando tiene un interés muy especial en sí mismo» (p. 11). El estudio se llevó a cabo en la ciudad de Cartagena durante el curso académico 2013-2014, en la Institución Educativa Ana María Vélez de Trujillo ${ }^{3}$, sede Loma Fresca. Allí se tomaron muestra de 15 estudiantes de los grados $3^{\circ}, 4^{\circ}$ y $5^{\circ}$ de la básica primaria (jornada vespertina), tres profesores de los respectivos grados y 15 padres de familia. A todos se les realizaron entrevistas y encuestas, llevándose a cabo además observación directa, grupos de discusión y análisis documental. La investigación se llevó a cabo en tres fases: 


\section{Recolección y descripción de la información}

Se inició con el proceso de formulación de la propuesta, diseño, planificación y selección de técnicas e instrumentos pertinentes. Luego se llevó a cabo una observación directa del entorno para caracterizar la población. Para ello se acudió a fichas que contienen la descripción de aspectos relevantes para la identificación y descripción de las prácticas pedagógicas de los docentes, abordadas desde las dimensiones de planeación, ejecución y evaluación.

La técnica de observación participante fue valiosa a la hora de recabar información acerca de la dinámica del aula en la interacción maestro-aprendiz. Como anota De Tezanos (2004), «la observación es la manera más fidedigna posible de las representaciones que el otro o los otros construyen sobre el vínculo que las personas observadas establecen con su entorno y entre ellas mismas» (p. 86). La observación tuvo lugar en diferentes momentos (actos cívicos, comunidades al inicio de la jornada escolar o salidas de campo), lo que permitió sumergir a las investigadoras en tales situaciones con el objeto de interpretar y analizar los hechos.

En la dinámica del aula, el maestro debe hacer mayor despliegue del saber pedagógico en su mediación entre el aprendizaje y el conocimiento, así como del uso de recursos, ambiente áulico, contextualización de las temáticas, y la práctica pedagógica desarrollada en atención a los estilos y ritmos de aprendizaje de los estudiantes. La observación directa permitió analizar con mayor profundidad las situaciones del aula, lo que llevó a detectar que el esquema educativo se enmarca en lo tradicional y rutinario, dejando ver en este sentido la necesidad de dotar de nuevos significados a la práctica pedagógica para hacer del aprendizaje algo atrayente y significativo.

El grupo de discusión permitió extraer información relevante de los sujetos de estudio, pues fue posible determinar puntos de encuentro así como también de divergencia en cuanto al desarrollo de su práctica. Se pudieron evidenciar las tensiones que surgen cuando al maestro se lo lleva a la reflexión sobre la práctica pedagógica, pues tiende a considerar que son los estudiantes los que presentan barreras para aprender. Sin embargo, se llegó a consensos sobre la importancia de innovar, recrear y analizar la propia práctica como principio para empezar su transformación, evitando que esta se constituya en una barrera para el aprendizaje.

Por último, el análisis documental del plan de estudio, del plan de clases, del observador del estudiante, de actas de promoción y evaluación por periodos, además de la implementación de la entrevista semiestructurada y la encuesta, facilitaron recabar información de gran valor que permitió determinar que la inclusión solo es un proceso posible si se permea cada estamento y cada acción educativa en pro de la misma.

\section{Categorización y análisis de la información}

La información recolectada se organizó en categorías para su posterior análisis, descomponiéndose luego en unidades temáticas afines que se agruparon o asociaron de acuerdo a su naturaleza con el fin descubrir las relaciones entre ellas. Se determinaron tres categorías a saber:

a Prácticas pedagógicas: resignificación del saber: Esta categoría recoge los resultados obtenidos orientados a describir las prácticas pedagógicas ejecutadas por los docentes de básica primaria (grados tercero, cuarto y quinto de la Institución Educativa Ana María Vélez de Trujillo, sede Loma Fresca, jornada vespertina) para favorecer la inclusión educativa, las percepciones acerca de la práctica pedagógica, y sus implicaciones en el proceso de enseñanza y aprendizaje.

b Didácticas flexibles: construyendo caminos, posibles rutas: En esta categoría se hace referencia a las estrategias metodológicas y didácticas que en el desarrollo de su quehacer pedagógico los maestros emplean para favorecer la atención a la diversidad. 
c Inclusión educativa: un horizonte de posibilidades: En esta categoría se abordaron las percepciones y conceptualizaciones manejadas por los maestros acerca de la inclusión educativa, así como la forma de favorecerla a partir de sus sentires y saberes.

\section{$3 \quad$ Interpretación y teorización}

A partir de la triangulación hermenéutica se corroboró la validez y confiabilidad de los datos obtenidos. El producto de esta labor es el informe final donde se exponen conclusiones y recomendaciones pertinentes a los hallazgos.

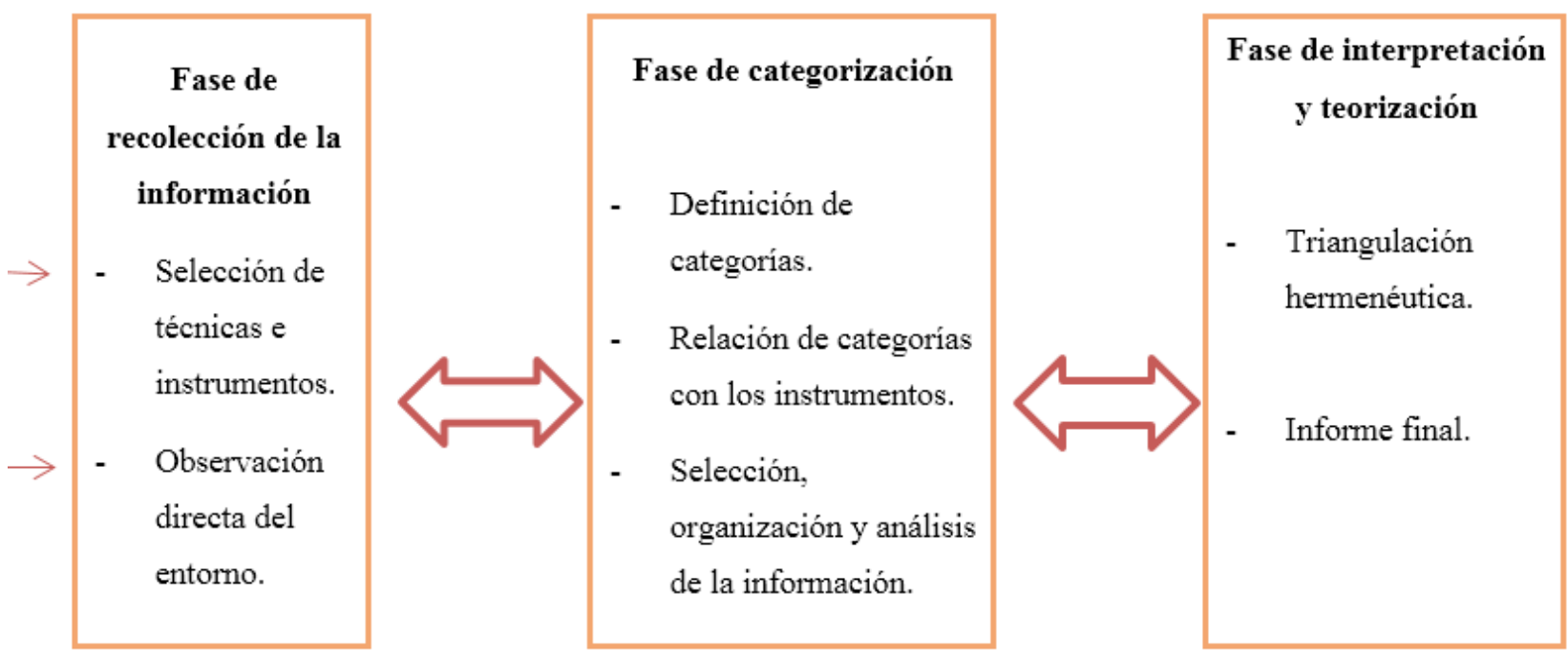

Figura 1. Fases de la investigación.

Cerrando el círculo hermenéutico, es pertinente para las investigadoras resaltar aportes significativos desde las voces de los implicados.

El camino de mejora educativa comienza con la reflexión que hagan los maestros sobre su práctica, pues una visión más reflexiva suscitará en ellos el deseo de fortalecer su quehacer. La articulación de las didácticas flexibles con las prácticas pedagógicas para favorecer la inclusión en la escuela está supeditada fundamentalmente al cambio de actitudes y estilos de enseñanza de los docentes. Supone esta nueva forma de ver el aprendizaje un mayor compromiso, disposición y apertura para trabajar colaborativamente para así poder aproximarse a una práctica reflexiva que se posiciona bajo otro paradigma: la aceptación y valoración de la diversidad en el aula.

Frente a este compromiso docente surgen otros elementos que optimizan el proceso, como es la toma de conciencia del valor de la diversidad desde todos los estamentos de la comunidad educativa: familias, estudiantes, docentes y directivos docentes. Los estudiantes y padres de familia consideran que la escuela debe propiciar actividades culturales, deportivas y recreativas con la intención de desarrollar habilidades, intereses y promover la aceptación y valoración de la diferencia. La propuesta de desarrollo curricular inclusiva aplicando el DUA ha sido una experiencia pedagógica valiosa producto de la reflexión de los docentes en el grupo de discusión y en la cual se evidenció que es posible propiciar nuevas formas de enseñar, donde docentes, estudiantes y padres de familia le apuestan al cambio. 
Resignificación de la práctica pedagógica

\section{Resultados}

Los resultados obtenidos en la presente investigación se organizaron por categorías con sus respectivos indicadores e ítems para su análisis.

\section{Prácticas Pedagógicas: resignificación del saber}

La entrevista aplicada a los docentes permitió indagar sobre cómo conciben y perciben la práctica pedagógica para favorecer la inclusión educativa, examinando aspectos como si la planeación atiende al contexto, el tipo de recurso usado, si las temáticas responden a los intereses y necesidades de los estudiantes, la preparación del ambiente escolar, la evaluación como proceso para aprender, etc. Con ello se pudo determinar que la práctica pedagógica es homogeneizante y basada en el tradicionalismo que invisibiliza las diferencias individuales, desconociendo que la educación debe ser contextualizada, por cuanto es educación para la vida.

También se identificó que existe un desfase entre el discurso y la práctica. Los docentes se preocupan por cómo articular lo que se debe enseñar con los intereses de los aprendices, pero se privilegian los contenidos preestablecidos en los planes de clases. Así se infiere de lo expresado por el Docente 2 (D2): «Sí me interesa qué quieren aprender mis estudiantes, aunque se deba sacar tiempo extra que sale de descontar tiempo a las horas de clases planeadas».

En la dinámica de la entrevista, se reconoce que la formación permanente es fundamental para propiciar en las prácticas pedagógicas cambios de posturas, reflexionando sobre cómo estas inciden en nuevas oportunidades para que todos los aprendices, desde sus limitaciones y posibilidades, accedan al conocimiento. La situación es reconocida por el MEN (2008), cuando expresa que «los docentes se enfrentan a un reto importante y es cambiar el esquema de homogeneidad por el de heterogeneidad» (p. 28). En este sentido, al hablar de prácticas pedagógicas se hace referencia a la forma de trabajo propia de cada docente en el aula donde va a intervenir su formación profesional, el contexto, así como su propia forma de aprender, lo que indiscutiblemente va a influenciar el desarrollo de sus prácticas pedagógicas y el discurso mismo.

\section{Inclusión educativa: un horizonte de posibilidades}

El sentir de los maestros con respecto a los procesos inclusivos en la escuela es el de reconocimiento del valor del otro y de acogida en términos generales. Sin embargo, a pesar de que manejan algunos conceptos relacionados con la inclusión como proceso y reconocen que la población estudiantil que accede a las aulas es diversa, manifiestan la necesidad de recibir capacitación para atender a esa diversidad. Desde el discurso se aprecia la disposición de los maestros, pero en la práctica los aprendices son los que deben adaptarse al ambiente escolar. Al respecto, el Docente 3 (D3) indica: «La inclusión educativa en la institución no la veo muy clara, pues los estudiantes y algunos docentes rechazan fuertemente a aquellos niños o niñas que vienen con alguna diferencia física o intelectual». Asimismo, si bien reconocen la atención a la diversidad como una realidad, la perciben como una barrera y un obstáculo. A propósito, el Docente 1 (D1) explica: «Pienso que debemos prepararnos más para manejar esa diversidad escolar ya que es difícil porque algunos niños necesitan atenciones especiales que ayuden a superar sus limitaciones». Como lo indica este testimonio, persisten en los maestros imaginarios que asocian la diversidad con necesidades educativas especiales, pues se percibe la inclusión educativa como educación especial, desconociendo todo lo que en su esencia implica el concepto. 


\section{Didácticas flexibles: construyendo caminos, posibles rutas}

La última categoría entreteje nuevas posibilidades de favorecer mejores ambientes de aprendizaje a partir de prácticas mediadas por las didácticas flexibles. En este sentido, los docentes reconocen que existen nuevos caminos y posibles rutas para que los aprendices accedan al conocimiento, independientemente de sus capacidades, contextos o experiencias personales, eliminando las barreras físicas, sensoriales, cognitivas y afectivas para el aprendizaje y la participación de los escolares. Los maestros consideran que las didácticas flexibles ofrecen la posibilidad de atención a la diversidad por cuanto permiten que se tengan en cuenta y atiendan los diferentes estilos cognitivos y ritmos de aprendizaje de los aprendices. Al respecto, los Docentes 3 y 1 observan:

Las didácticas flexibles ayudan a los estudiantes a integrarse más al proceso de aprendizaje ya que se hace más dinámico y las actividades lúdicas los ayudan en su proceso de adaptación a su ambiente escolar y a sus habilidades a través de juegos, actividades deportivas y otros (D3).

Las didácticas flexibles son aquéllas que se amoldan a las necesidades y actitudes de los estudiantes, estas nos ayudan a manejar de forma más fácil la diversidad de aprendizajes que encontramos en las aulas, esta busca facilitar el aprendizaje de todos los estudiantes teniendo en cuenta los conocimientos previos de ellos (D1).

De acuerdo con los resultados obtenidos en la encuesta aplicada a los padres de familia, es evidente que su mayor preocupación se centra en la gestión administrativa y de la comunidad especialmente en lo concerniente a la proyección de la institución en actividades lúdico-recreativas y de esparcimiento cultural-, así como la adecuación de la planta física y en los aportes significativos al proceso investigativo, pues dentro de las dimensiones de la inclusión educativa, la política y la cultura inclusiva se tocan, pero evidencian debilidades. Tales resultados deben mostrarse como base para la ruta a seguir en los planes de mejoramiento institucional. Los padres de familia (PF) develan la necesidad de brindar a sus hijos un ambiente más acogedor en donde no solo lo académico sea lo fundamental sino también el juego, el deporte y la recreación, como actividades necesarias para que los niños aprendan y tengan una mejor convivencia.

«La escuela debe mejorar. Los salones están muy apretados» (PF 3).

«La escuela debe mejorar. Se ve descuidada» (PF 5).

«Los maestros deben preocuparse por mejorar la escuela y realizar otras actividades para que nuestros hijos le tengan más amor a este lugar» (PF 1).

Una fortaleza de la institución es que admite a toda la población del sector, sin discriminación de raza, cultura, género, credo, ideología, condición socioeconómica o situaciones de vulnerabilidad como desplazamiento, extraedad, entre otras. Hay conocimiento por parte de la comunidad educativa sobre el proceso de enseñanza de los docentes; sin embargo, se hace necesario ofrecer más opciones didácticas que faciliten el aprendizaje y atiendan las necesidades educativas de estudiantes que en algunas ocasiones lo ameritan. Se observa cómo los padres de familia se sienten importantes cuando se les valora y se les reconoce como pilares fundamentales en el proceso de educación.

Los resultados obtenidos de la entrevista con los estudiantes develan que se debe afianzar el enfoque metodológico en la gestión académica para que permita al estudiante aprender colaborativamente teniendo en cuenta sus modos particulares de aprender. Igualmente, la planeación y desarrollo de 
las clases debe responder a sus intereses y necesidades. En este sentido, las actitudes y estilos de enseñanza de los docentes pueden contribuir a asegurar el éxito de más niños en el aula de clases y potenciar al máximo los aprendizajes. Pasar de prácticas homogeneizantes a prácticas que consideran la diversidad supone mirar la enseñanza a través de una actitud abierta, flexible y reflexiva por parte del docente para que se dé cuenta que mediante la aplicación de didácticas flexibles se puede garantizar la participación y potenciar al máximo los aprendizajes. Como lo afirma Delors (1996), opciones como elegir materiales, herramientas, contenidos, entre otros, así como ambientes libres de amenazas, distracciones o violencia, incrementan el aprendizaje, y vista así: «La educación puede ser un factor de cohesión social si procura tener en cuenta la diversidad de los individuos y de los grupos humanos, y al mismo tiempo, evitar ser a su vez un factor de exclusión social» (p. 59).

La escuela juega un papel fundamental en procura de la diversidad al favorecer la aceptación y valoración de las diferencias para accionar la premisa de aprender a ser y a vivir juntos, comprendiendo y valorando al otro como ser legítimo y válido. Siguiendo los planteamientos de Freire (2002), «la educación es un acto de amor, de coraje, es una práctica de la libertad dirigida hacia la realidad a la que no teme; más bien busca transformarla por solidaridad, por espíritu fraternal» (p. 30).

Las prácticas docentes que median ambientes de aprendizaje en donde la implementación de diversas estrategias posibiliten rutas diferentes para potenciar los aprendizajes, favorecen la inclusión en la institución. Así lo refleja el testimonio del Estudiante 1 (E1): «Me gustaría hacer otras actividades en clases, hacer más juegos y actividades deportivas, trabajar con computadores y hacer salidas fuera del colegio».El plan de estudio institucional sigue caracterizado por un enfoque tradicional de la educación que la fragmenta por asignaturas, desintegrando también la visión de la realidad y el conocimiento. Es pertinente entonces que se reflexione sobre el plan, ya que debe abordarse como un todo integrador y direccionador de la labor docente. De ahí que surja la necesidad de planearlo teniendo en cuenta que debe favorecer el desarrollo de las dimensiones del ser en aras de lograr un desarrollo integral con profundo sentido humano, donde lo que se enseñe sea contextualizado, significativo y pertinente para el aprendiz.

Los resultados obtenidos a partir de la aplicación de las técnicas investigativas concernientes a articular las didácticas flexibles con las prácticas pedagógicas para favorecer la inclusión en la escuela están supeditados fundamentalmente al cambio de actitudes y estilos de enseñanza de los docentes. Esta nueva forma de ver el aprendizaje supone de mayor compromiso, disposición y apertura para trabajar colaborativamente y de esta manera poder aproximarse a una práctica reflexiva que se posiciona bajo otro paradigma: la aceptación y valoración de la diversidad en el aula.

Este estudio evidencia la importancia de la práctica pedagógica como facilitadora de los procesos inclusivos en cualquier contexto educativo, pero especialmente en aquellos de vulnerabilidad social en los que es preciso avanzar hacia una oferta educativa de mejor calidad que rompa con los imaginarios y estereotipos que consideran, en la mayoría de los casos, que un aprendiz de estos contextos no logrará posicionarse fácilmente en la sociedad como un ser humano útil, capaz de transformarse y transformar su entorno.

Es evidente la multiplicidad de modos y formas de lograr la formación de mejores ciudadanos y personas con alto sentido y calidad humana. Sin embargo, como motor de cambio, la educación debe asumir un papel más determinante. De allí que si reconocemos que la responsabilidad de la enseñanza recae principalmente en los maestros, estos deben avanzar hacia la mejora continua y sistemática de su práctica. Los resultados arrojados por este estudio ponen sobre el tapete la relevancia de la práctica pedagógica reflexiva como potenciadora de avances significativos para mejorar la calidad educativa actual y como facilitadora no solo del acceso sino de la permanencia de los aprendices en el contexto 
educacional, toda vez que se llenan sus expectativas e intereses.

La implementación de didácticas flexibles aporta elementos de peso para considerar la ejecución de otros estudios donde entren en juego el despliegue de innovaciones y otras didácticas (como la geempiana) que favorecen los procesos de alfabetización, especialmente en contextos de vulnerabilidad social.

\section{Conclusiones y recomendaciones}

Teniendo en cuenta los objetivos de la presente investigación y después de haber analizado e interpretado los datos obtenidos en la misma, se esbozan las siguientes conclusiones. Al momento de describir las prácticas pedagógicas de los maestros se encuentra que se desarrollan bajo un modelo de educación tradicional no acorde con lo expuesto en el Proyecto Educativo Institucional (PEI), el cual se enmarca en principios inclusivos que procuran una enseñanza que considera la diversidad como valor fundamental. Así, existe poca apropiación por parte de los maestros acerca del modelo existente.Cuando se indagó sobre las percepciones y concepciones sobre la inclusión educativa, la perciben como un proceso impuesto por la institución para el cual no han sido capacitados, lo que les impide abordarla de manera más asertiva desde el aula. Por ello los maestros consideran la inclusión educativa como un reto por asumir.

Desde los hallazgos y experiencias de estrategias didácticas utilizadas por los docentes para favorecer la inclusión educativa, se encuentra que implementan algunas actividades que a su juicio favorecen la inclusión de manera aislada. Así pues, no existe un plan de trabajo direccionado para favorecer este aspecto y los contenidos se imparten del mismo modo y con las mismas estrategias pedagógicas, situación que encuentra justificación en la falta de capacitación docente. En consecuencia, los docentes no miran la diversidad como valor sino como sinónimo de dificultades de aprendizaje.

Al interior del grupo de maestros se evidenció una actitud abierta y dispuesta para la implementación de nuevas didácticas que favorezcan el proceso de inclusión educativa, toda vez que se reflexionó sobre las prácticas actuales y sus posibilidades de articulación con las didácticas flexibles. Tal disposición repercutió en el logro de un mejor proceso de enseñanza aprendizaje, dejando como insumo de experiencias de aula dos propuestas de desarrollo curricular inclusivo a partir del DUA.

Cuestionar y reflexionar sobre el quehacer educativo no es sencillo, pues resulta fácil desestabilizar a los docentes a partir del cuestionamiento de su propia práctica cuando no se les proponen nuevos caminos y posibles rutas que los orienten en su accionar pedagógico. A partir de los resultados arrojados en este estudio es pertinente, a juicio de las investigadoras, plantear algunas recomendaciones con el ánimo de contribuir al mejoramiento de la calidad educativa que se ofrece, no solo en la Institución Educativa Ana María Vélez de Trujillo, sino en todo el Caribe colombiano y el país entero.

En virtud de lo anterior se recomienda que los maestros se interesen por investigar sobre su propia práctica, en una praxis reflexiva que se construya y deconstruya permanentemente con el ánimo de la mejora continua. Los maestros deben también conocer las características del contexto social en el que se encuentran inmersos sus aprendices y todas las dimensiones que esto involucra a nivel familiar, social, cultural, económico y político, ya que esto les permitirá direccionar los contenidos y utilizar una pedagogía apropiada que, además de atender a la diversidad de los estudiantes, provoque en ellos el interés por aprender y comprender cómo funciona la sociedad, cómo resolver los problemas de la misma, y lo que es más importante, reconocer cuál es su papel en el mundo.

Asimismo, se recomienda formar grupos de aprendizaje colaborativos con representantes de cada una de las sedes de la institución para que en conjunto trabajen en el intercambio de experiencias significativas y logren una retroalimentación permanente. También es relevante gestionar la 
capacitación docente en la Secretaría de Educación Distrital y en la administración de la institución educativa, a través de un diplomado sobre inclusión educativa y didácticas flexibles.

La institución educativa debe trabajar en replantear la política institucional atendiendo al proceso de inclusión educativa, pues no basta solo con acoger a una población diversa. Por tal motivo se hace necesario trazar acciones claras y emprender las adaptaciones curriculares que la política de inclusión nacional promueve.

Finalmente, es clave continuar propiciando escenarios de diálogo reflexivo con los estudiantes y padres de familia acerca de situaciones escolares, para que a partir de sus aportes constructivos se den posibles respuestas a las necesidades educativas y sociales del contexto donde está inmersa la escuela.

\section{Notas}

${ }^{1}$ Ofrecer diferentes opciones para abordar los contenidos temáticos dando posibilidades para la comprensión.

${ }^{2}$ Ofrecer variadas opciones por medio de diferentes recursos materiales para facilitar la expresión y la fluidez.

${ }^{3}$ La institución, de carácter oficial, se encuentra ubicada en la zona norte de Cartagena en el barrio Torices, Santa Rita, con sede en las faldas de la Popa. Atiende población de estratos 0 y 1, de preescolar a secundaria.

\section{Referencias}

Arana G, G. M. (2012). Plan de capacitación a docentes para lograr el aprendizaje significativo en los estudiantes de la unidad básica Mons. Juan Wiesneth Rural del cantón Naranjito. Ecuador (Tesis de Maestría). Universidad Estatal del Milagro, Milagro, Ecuador.

Arias B, L. T., Bedoya A, K., Benítez P, C., Carmona C, L. Y., Castaño Ú, J. C., Castro G, L. M., Pérez C, L. Y. \& Villa B, L. M. (2007). Formación docente: una propuesta para promover prácticas pedagógicas inclusivas. Revista Educación y Pedagogía, Universidad de Antioquia, vol. XIX (47), ene-abr, pp. 153-162.

Blanco G, R. (2006). La equidad y la inclusión social: Uno de los desafíos de la Educación y la Escuela Hoy. REICE. Revista Iberoamericana sobre Calidad, Eficacia y Cambio en Educación, 4(3), pp. $1-15$.

Booth, T., \& Ainscow, M. (2000). Índice de Inclusión. Bristol, UK: Centre for studies in inclusive education.

Center for Applied Special Technology (CAST). (2011). Universal design learning guidelines versión 2.0. Wakefield, MA: Author. Traducido al español por Universidad Complutense de Madrid, $2-5$.

De Tezanos, A. (1998). Una etnografía de la etnografía. Bogotá: Antropos.

Díaz H, O. D. C., Franco M, F. R., \& Tovar G, J. A. (2009). Percepción y actitud de los docentes hacia la inclusión educativa en soledad, 2008 (Disertación doctoral).

Erickson, F. (1989). Métodos cualitativos de investigación sobre la enseñanza. Madrid: Paidos-MEC Freire, P. (2005). Pedagogía del oprimido. Barcelona: Siglo XXI.

Gardner, H. (1993). Inteligencias múltiples: La teoría de la práctica. Buenos Aires: Paidos.

Giné, C. G., \& Font, J. (2007). El alumnado con discapacidad intelectual y del desarrollo. En J. B. Picas y M. Sanchez (Eds.), Manual de asesoramiento psicopedagógico (pp. 879-914). Barcelona: Graó.

Gisbert, D. D., \& Giné, C. G. (2011). La formación del profesorado para una educación inclusiva: 
Un proceso de desarrollo profesional y de mejora de los centros para atender a la diversidad. Revista Latinoamericana de Educación Inclusiva, 5(2), pp. 153-170.

Hopkins, D., \& Stern, D. (1996). Quality teachers, quality school: International perspectives and policy implications. Teaching and teacher education, 12(5), p. 501. DOI: http://dx.doi. org/10.1016/0742-051x(95)00055-o.

Imbernón, F. (1994). La formación y el desarrollo profesional del profesorado: hacia una cultura profesional. Barcelona: Graó Delors, J. (1996). La educación encierra un tesoro. Madrid: Santillana - Unesco.

Larrivee, B. (2000). Transforming teaching practice: Becoming the critically reflective teacher. Reflective practice, 1(3), pp. 293-307. DOI: http://dx.doi.org/10.1080/713693162.

López V, A. L. (2008). Fomentando la reflexión sobre la atención a la diversidad. REICE. Revista Iberoamericana sobre Calidad, Eficacia y Cambio en Educación.

Mejía, H., \& Molina, A. (2012). Estrategias pedagógicas inclusivas para la estimulación del desarrollo de competencias afectivas. Magdalena.

Ministerio de Educación Nacional (MEN). (2008). Guía de educación inclusiva. Bogotá.

Montolio P, R., \& Cervellera M, L. (2008). Una escuela de todas (las personas) para todas (las personas). España.

Moriña-D, A. (2004). Teoría y práctica de la educación inclusiva. Málaga: Ediciones Aljibe.

Ortiz, C. (2000). Hacia una educación inclusiva. La educación especial ayer, hoy y mañana. Siglo Cero, 31(1), pp. 5-11.

Rodrigues, R. (2013). El desarrollo de la práctica reflexiva sobre el que hacer docente, apoyada en el uso de un portafolio digital, en el marco de un programa de formación para académicos de la Universidad Centroamericana de Nicaragua. (Tesis Doctoral). Universidad de Barcelona, Barcelona, España.

Rosano O, S. (2008). El camino de la inclusión educativa en Punta Hacienda (comunidad campesina de la sierra andina ecuatoriana). (Tesis de Maestría). Universidad Internacional de Andalucía, Sevilla, España.

Ruiz O, J. I. (1996). Metodología de la investigación cualitativa. Bilbao: Universidad de Deusto, 217.

Stake, R. E. (1998). Investigación con estudio de casos. Madrid: Ediciones Morata, 11.

Unesco. (2005). El imperativo de la calidad. Informe de seguimiento de educación para todos en el mundo. París: Unesco.

Vygotsky, L. (1989). El desarrollo de los procesos psicológicos superiores. Barcelona: Crítica. 